\title{
Vector Partitions and Combinatorial Identities
}

\author{
By M. S. Cheema
}

In this note we show how certain relations between vector partition functions can be deduced from certain identities. A relation connecting vector partitions having odd components and those having distinct parts will be proved. A combinatorial proof of Jacobi's Identity similar to Franklin's proof of Euler identity is suggested. The last section includes numerical values of $P_{r}(n, m)$ and $q_{r}(n, m)$. These results suggest the unique maxima property of $P_{r}(n, m)$ for fixed $n, m$ and $r$ varying.

In the Jacobi Identity

$$
\prod_{n=1}^{\infty}\left(1-q^{2 n}\right)\left(1+q^{2 n-1} t\right)\left(1+q^{2 n-1} t^{-1}\right)=\sum_{-\infty}^{+\infty} q^{n^{2}} t^{n}
$$

make the substitution $q^{2}=x y, t^{2}=x / y$ and change $x$ to $-x, y$ to $-y$ to obtain

$$
\prod_{n=1}^{\infty}\left(1-x^{n} y^{n}\right)\left(1-x^{n} y^{n-1}\right)\left(1-x^{n-1} y^{n}\right)=\sum_{-\infty}^{+\infty}(-1)^{n} x^{n(n+1) / 2} y^{n(n-1) / 2}
$$

This when interpreted combinatorially yields the following

Theorem I. The excess of the number of partitions of $(n, m)$ into even number of distinct parts of the type $(a, a-1),(b-1, b),(c, c)$ over those into odd number of such parts is $(-1)^{r}$ or 0 according as $(n, m)$ is of the type $(r(r+1) / 2, r(r-1) / 2)$ or not.

Let $\alpha(n, m)$ denote the number of partitions of $(n, m)$ into distinct parts $(a, a-1),(b-1, b)$ so that we have the generating function

$$
\sum_{n, m=0}^{\infty} \alpha(n, m) x^{n} y^{m}=\prod_{n=1}^{\infty}\left(1+x^{n} y^{n-1}\right)\left(1+x^{n-1} y^{n}\right)
$$

In 1.1 making the substitution $q^{2}=x y, t^{2}=x / y$ we obtain

$$
\begin{aligned}
\prod_{n=1}^{\infty}\left(1+x^{n} y^{n-1}\right)\left(1+x^{n-1} y^{n}\right) & =\left\{\prod_{n=1}^{\infty}\left(1-x^{n} y^{n}\right)\right\}^{-1}\left\{\sum_{-\infty}^{+\infty} x^{n(n+1) / 2} y^{n(n-1) / 2}\right\} \\
& =\left\{\sum_{n=1}^{\infty} p(n) x^{n} y^{n}\right\}\left\{\sum_{-\infty}^{+\infty} x^{n(n+1) / 2} y^{n(n-1) / 2}\right\} .
\end{aligned}
$$

Equating coefficients Carlitz [2] obtained

$$
\alpha(n, m)=p\left(n-\frac{1}{2}(n-m)(n-m+1)\right) .
$$

Conversely if one can prove this result combinatorially it yields a proof of Jacobi's Identity, such a proof has been obtained by Wright in a forthcoming paper by setting up a 1-1 correspondence between the two types of partitions.

This is done by placing a triangular array of $(n-m)(n-m+1) / 2$ dots on the graph of each partition of $n-\frac{1}{2}(n-m)(n-m+1)$, the columns under the diagonal and rows on the right side determine uniquely parts $(a, a-1),(b-1, b)$ of $(n, m)$.

Received November 18, 1963. Revised January 30, 1964. Research supported in part by N.S.F. Grant number 575-503. 
If one can prove Theorem I by combinatorial arguments similar to Franklin's proof of Euler identity

$$
\prod_{r=1}^{\infty}\left(1-x^{r}\right)=\sum_{-\infty}^{+\infty}(-1)^{\lambda} x^{\lambda(3 \lambda+1) / 2},
$$

it will yield a combinatorial proof of Jacobi's Identity. The method of proof will depend on setting up a 1-1 correspondence between the partitions into even number of distinct parts and odd number of distinct parts of the type $(a, a-1),(b-1, b)$, $(c, c)$; such a correspondence has to be 1-1 both ways. By means of simple operations one can change the parity of the number of parts except in the case when $(n, m)$ is of the type $(r(r+1) / 2, r(r-1) / 2)$, the parity of whose partition $(r, r-1),(r-1, r-2), \cdots,(2,1),(1,0)$ cannot be changed and thus the excess in this case is $(-1)^{r}$ and 0 in other cases.

Gordon [1] has generalized Jacobi's Identity such that there are five products on the left side, i.e.,

$$
\begin{aligned}
\prod_{n=1}^{\infty}\left(1-q^{2 n}\right)\left(1-q^{2 n-1} t\right)\left(1-q^{2 n-1} t^{-1}\right)\left(1-q^{4 n-4} t^{2}\right)\left(1-q^{4 n-4} t^{-2}\right) \\
=\sum_{-\infty}^{+\infty} q^{3 n^{2-2 n}}\left(t^{3 n}+t^{-3 n}-t^{3 n-2}-t^{-3 n+2}\right) .
\end{aligned}
$$

Again put $q^{2}=x y, t^{2}=x / y$ to obtain

$$
\begin{aligned}
\prod_{n=1}^{\infty}( & \left.1-x^{n} y^{n}\right)\left(1-x^{n} y^{n-1}\right)\left(1-x^{2 n-1} y^{2 n-3}\right)\left(1-x^{2 n-3} y^{2 n-1}\right)\left(1-x^{n-1} y^{n}\right) \\
=\sum_{n=-\infty}^{+\infty}\left\{x^{\left(3 n^{2}+n\right) / 2} y^{\left(3 n^{2}-5 n\right) / 2}+x^{\left(3 n^{2}-5 n\right) / 2} y^{\left(3 n^{2}+n\right) / 2}\right. & -x^{\left(3 n^{2}+n-2\right) / 2} y^{\left(3 n^{2}-5 n+2\right) / 2} \\
& \left.-x^{\left(3 n^{2}-5 n+2\right) / 2} y^{\left(3 n^{2}+n-2\right) / 2}\right\} .
\end{aligned}
$$

Let $C(c, m)$ denote number of partitions of $(n, m)$ into vectors of the type $(a, a)$, $(b, b-1),(c-1, c),(2 d-1,2 d-3),(2 e-3,2 e-1)$; thus the generating function is given by

$$
\begin{array}{r}
\prod_{n=1}\left\{\left(1-x^{n} y^{n}\right)\left(1-x^{n} y^{n-1}\right)\left(1-x^{n-1} y^{n}\right)\left(1-x^{2 n-1} y^{2 n-3}\right)\left(1-x^{2 n-3} y^{2 n-1}\right)\right\}^{-1} \\
=\sum_{n, m=0}^{\infty} C(n, m) x^{n} y^{m} .
\end{array}
$$

Thus (1.6) yields the recurrence relation

$$
\begin{aligned}
\sum C\left(n-\frac{3 r^{2}+r}{2},\right. & \left.m-\frac{3 r^{2}-5 r}{2}\right)+\sum C\left(n-\frac{3 r^{2}-5 r}{2}, m-\frac{3 r^{2}+r}{2}\right) \\
& -\sum C\left(n-\frac{3 r^{2}+r-2}{2}, m-\frac{\left(3 r^{2}+5 r+2\right.}{2}\right) \\
& -\sum C\left(n-\frac{\left(3 r^{2}-5 r+2\right)}{2}, m-\frac{\left(3 r^{2}-r-2\right)}{2}\right)=0 .
\end{aligned}
$$

Change $x$ to $-x, y$ to $-y$ in (1.5) to obtain 


$$
\begin{aligned}
& \prod_{n=1}^{\infty}\left(1-x^{n} y^{n}\right)\left(1+x^{n} y^{n-1}\right)\left(1+x^{n-1} y^{n}\right)\left(1-x^{2 n-1} y^{2 n-3}\right)\left(1-x^{2 n-3} y^{2 n-1}\right) \\
& \quad=\sum_{n=-\infty}^{+\infty}(-1)^{n} x^{\left(3 n^{2}+n\right) / 2} y^{\left(3 n^{2}-5 n\right) / 2}+\sum_{-\infty}^{+\infty}(-1)^{n} x^{\left(3 n^{2}-5 n\right) / 2} y^{\left(3 n^{2}+n\right) / 2} \\
& +\sum_{-\infty}^{+\infty}(-1)^{n+1} x^{\left(3 n^{2}+n-2\right) / 2} y^{\left(3 n^{2}-5 n+2\right) / 2}+\sum_{-\infty}^{+\infty}(-1)^{n+1} x^{\left(3 n^{2}-5 n+2\right) / 2} y^{\left(3 n^{2}+n-2\right) / 2}
\end{aligned}
$$

If $D(n, m)$ denotes the number of partitions of $(n, m)$ into parts of the type $(2 d-1,2 d-3),(2 e-3,2 e-1)$. We obtain a relation between $\alpha(n, m)$ and $D(n, m)$ by writing $(1.8)$ in the form

$$
\begin{aligned}
&\left\{\sum(-1)^{\lambda}(x y)^{\lambda(3 \lambda+1) / 2}\right\}\left\{\bigcup_{n, m=0}^{\infty} \alpha(n, m) x^{n} y^{m}\right\} \\
&=\left\{\sum_{n, m=0}^{\infty} D(n, m) x^{n} y^{m}\right\}\left\{\sum(-1)^{n} x^{\left(3 n^{2}+n\right) / 2} y^{\left(3 n^{2}-5 n\right) / 2}\right. \\
&+(-1)^{n} x^{\left(3 n^{2}-5 n\right) / 2} y^{\left(3 n^{2}+n\right) / 2}+(-1)^{n+1} x^{\left(3 n^{2}+n-2\right) / 2} y^{\left(3 n^{2}-5 n+2\right) / 2} \\
&\left.+(-1)^{n+1} x^{\left(3 n^{2}-5 n+2\right) / 2} y^{\left(3 n^{2}+n-2\right) / 2}\right\}
\end{aligned}
$$

and equating coefficients.

1.5 can also be written as

$$
\begin{aligned}
\sum_{-\infty}^{+\infty}(-1)^{n} x^{n(n+1) / 2} y^{n(n-1) / 2}=\left\{\sum_{n, m=0}^{\infty} D(n, m) x^{n} y^{m}\right\} & \cdot\left\{\sum_{-\infty}^{\infty} x^{\left(3 n^{2}+n\right) / 2} y^{\left(3 n^{2}-5 n\right) / 2}+\sum_{-\infty}^{\infty} x^{\left(3 n^{2}-5 n\right) / 2} y^{\left(3 n^{2}+n\right) / 2}\right. \\
& \left.-\sum_{-\infty}^{+\infty} x^{\left(3 n^{2}+n-2\right) / 2} y^{\left(3 n^{2}-5 n+2\right) / 2}-\sum x^{\left(3 n^{2}-5 n+2\right) / 2} y^{\left(3 n^{2}+n+2\right) / 2}\right\},
\end{aligned}
$$

equating coefficients

$$
\begin{aligned}
\sum D(n & \left.-\frac{3 r^{2}+r}{2}, m-\frac{3 r^{2}-5 r}{2}\right) \\
& +\sum D\left(n-\frac{3 r^{2}-5 r}{2}, m-\frac{3 r^{2}+r}{2}\right) \\
& -\sum D\left(n-\frac{3 r^{2}-5 r+2}{2}, m-\frac{3 r^{2}+r-2}{2}\right) \\
& -\sum D\left(n-\frac{3 r^{2}+r-2}{2}, m-\frac{3 r^{2}-5 r+2}{2}\right)=(-1)^{r} \text { or } 0
\end{aligned}
$$

according as $(n, m)$ is of the type $(r(r+1) / 2, r(r-1) / 2)$ or not. The Jacobi Identity

$$
\prod_{n=1}^{\infty}\left(1-x^{n} y^{n}\right)\left(1+x^{n-1} y^{n}\right)\left(1+x^{n} y^{n-1}\right)=\sum_{-\infty}^{+\infty} x^{n(n+1) / 2} y^{n(n-1) / 2}
$$

can be written as

$$
\left\{\sum_{\lambda=0}^{\infty}(-1)^{\lambda}(x y)^{\lambda(3 \lambda \pm 1) / 2}\right\}\left\{\sum_{n, m=0}^{\infty} \alpha(n, m) x^{n} y^{m}\right\}=\sum_{-\infty}^{+\infty} x^{r(r+1) / 2} y^{r(r-1) / 2}
$$


Thus equating coefficients

$$
\sum_{\lambda}(-1)^{\lambda} \alpha\left(n-\lambda \frac{(3 \lambda \pm 1)}{2}, m-\lambda \frac{(3 \lambda \pm 1)}{2}\right)=1 \text { or } 0
$$

according as $(n, m)$ is or is not of the type $(r(r+1) / 2, r(r-1) / 2)$.

In the case of the number of partitions of an integer we have the well-known result that the number of partitions of $n$ into odd parts is equal to the number of partitions of $n$ into distinct parts. We can prove the following generalization of this result for vector partitions.

TheOREM II. The number of partitions of $\left(n_{1}, n_{2}, \cdots, n_{s}\right)$ into vectors with at least one component odd is equal to the number of partitions of $\left(n_{1}, n_{2}, \cdots, n_{s}\right)$ into distinct parts (vectors). Note, the same result holds if the parts are required to have non-zero components.

Proof. Denote the generating function of unrestricted vector partitions by

$$
\begin{aligned}
f\left(x_{1}, x_{2}, \cdots, x_{s}\right) & =\prod_{k_{i} \geqq 0}^{\infty}\left(1-x_{1}^{k_{1}} x_{2}{ }^{k_{2}} \cdots x_{s}{ }^{k_{s}}\right)^{-1} \\
& =\sum u\left(n_{1}, n_{2}, \cdots, n_{s}\right) x_{1}{ }^{{ }_{1}}, \cdots, x_{s}{ }^{{ }^{n_{s}}}
\end{aligned}
$$

and notice that the generating function for the number of partitions with at least one component odd is

$$
g\left(x_{1}, \cdots, x_{s}\right) \prod_{j_{i} \geqq 0}\left\{\left(1-x_{1}^{j_{1}}, x_{2}^{j_{2}} \cdots x_{8}^{j_{s}}\right)\right\}^{-1}
$$

where at least one $j_{i}$ is odd.

This is connected with $f\left(x_{1}, \cdots, x_{s}\right)$ by

$$
g\left(x_{1}, \cdots, x_{s}\right)=\frac{f\left(x_{1}, \cdots, x_{s}\right)}{f\left(x_{1}^{2}, \cdots, x_{s}^{2}\right)}=\prod_{k_{i} \geqq 0}\left(1+x_{1}^{k_{1}} x_{2}{ }^{k_{2}} \cdots x_{s}^{k_{s}}\right)
$$

and this proves the result.

Let

$$
\begin{aligned}
& f(x)=\left\{\prod_{n=1}^{\infty}\left(1-x^{n}\right)\right\}^{-1}, \\
& g(x)=\frac{f(x)}{f\left(x^{2}\right)^{2}}=\sum_{n=0}^{\infty} x^{n(n+1) / 2}, \\
& \theta(x)=\sum_{-\infty}^{+\infty} x^{n^{2}} .
\end{aligned}
$$

Gordon [1] has shown that

$$
\begin{aligned}
& F(x)=\frac{f\left(x^{2}\right) f\left(x^{3}\right)}{f(x)^{2} f\left(x^{6}\right)}=g(x)-3 x g\left(x^{9}\right), \\
& G(x)=\frac{f\left(x^{2}\right) f\left(x^{3}\right) f\left(x^{12}\right)}{f(x) f\left(x^{4}\right) f\left(x^{6}\right)^{2}}=\frac{3}{2} \theta\left(x^{9}\right)-\frac{1}{2} \theta(x) .
\end{aligned}
$$


Thus

$$
\begin{aligned}
\int_{0}^{1} x^{s^{2}-1} G(x) d x & =\frac{3}{2} \sum_{-\infty}^{+\infty} \frac{1}{9 n^{2}+s^{2}}-\frac{1}{2} \sum_{-\infty}^{+\infty} \frac{1}{n^{2}+s^{2}} \\
& =\frac{\pi}{2 s} \operatorname{Coth}\left(\frac{\pi s}{3}\right)-\frac{\pi}{2 s} \operatorname{Coth}(\pi s)
\end{aligned}
$$

when $s^{2} \rightarrow 0$

$$
\begin{aligned}
\int_{0}^{1}\{G(x)-1\} \frac{d x}{x} & =\frac{3}{2} \cdot 2 \sum_{1}^{\infty} \frac{1}{9 n^{2}}-\sum_{n=1}^{\infty} \frac{1}{n^{2}} \\
& =-\frac{2}{3} \cdot \frac{\pi^{2}}{6}=-\frac{\pi^{2}}{9} .
\end{aligned}
$$

Also

$$
\int_{0}^{1} x^{s^{2}}\{F(x)-1\} \frac{d x}{x}=\sum_{n=1}^{\infty} \frac{1}{\frac{n^{2}+n}{2}+s^{2}}-3 \sum_{n=1}^{\infty} \frac{1}{\frac{9 n^{2}+9 n+2}{2}+s^{2}}
$$

when $s^{2} \rightarrow 0$.

We obtain

$$
\int_{0}^{1}\{F(x)-1\} \frac{d x}{x}=2 \sum_{n=1}^{\infty} \frac{1}{n^{2}+n}-6 \sum_{n=1}^{\infty} \frac{1}{9 n^{2}+9 n+2}
$$

but $\sum_{n=1}^{\infty} 1 /\left(n^{2}+n\right)=1$. Thus

$$
\int_{0}^{1}\{F(x)-1\} \frac{d x}{x}=2-6 \sum_{n=1}^{\infty} \frac{1}{9 n^{2}+9 n+2} .
$$

The author recently extended the table of values $q_{r}(n, m)$ to $n, m=1(1) 49$, $r=1(1) 98$. These tables display the unique maxima property of $P_{r}(n, m)$ the number of partitions of $(n, m)$ into exactly $r$ parts with positive components. Szekeres [3] proved this result for $P_{r}(n)$ the number of partitions of $n$ into exactly $r$ parts. The value of $r=r_{0}$ for which such a maxima occurs was also obtained by Szekeres. It seems reasonable to conjecture that $P_{r}\left(n_{1}, n_{2}, \cdots, n_{s}\right)$ attains a unique maxima for fixed $n_{i}$ and $r$ varying, to locate the position of the maxima is still another problem, we hope these numerical results will be useful in establishing these results. Here we list the values of $q_{r}(n, m)$ and $P_{r}(n, m)$. The number of partitions of $(n, m)$ into a most $r$ parts and into exactly $r$ parts with positive components respectively for $n=m=49, r=1$ (1) 98. These calculations were performed on IBM7072 at the University of Arizona Computing Centre.

$\begin{array}{rrrrr}r & q_{r}(49,49) & r & P_{r}(49,49) \\ 1 & 1 & 1 & 1 \\ 2 & 1250 & 2 & 1152 \\ 3 & 271250 & 3 & 2 & 12352 \\ 4 & 20456138 & 4 & 125 & 40912 \\ 5 & 7253 & 82374 & 5 & 321383504 \\ 6 & 143784 & 40981 & 6 & 4255450133 \\ 7 & 1784775 & 75068 & 7 & 32701533936\end{array}$


$$
q_{r}(49,49)
$$$$
\begin{array}{lll}
150 & 48471 \quad 17166
\end{array}
$$$$
916 \quad 77113 \quad 62366
$$$$
42349821930204
$$$$
154737103936275
$$$$
461473945592382
$$

$\begin{array}{lllll}1 & 15673 & 58257 & 26397\end{array}$

$\begin{array}{lllll}2 & 49776 & 71698 & 61341\end{array}$

$4 \quad 7466054906 \quad 63150$

$808754 \quad 69334 \quad 51823$

$\begin{array}{lllll}12 & 55778 & 74241 & 16759\end{array}$

$\begin{array}{lllll}18 & 02318 & 70750 & 55031\end{array}$

24206682643928450

$\begin{array}{lllll}30 & 75237 & 65765 & 05129\end{array}$

$\begin{array}{lllll}37 & 29788 & 68910 & 41293\end{array}$

$\begin{array}{lllll}43 & 53218 & 32697 & 73604\end{array}$

$\begin{array}{llll}49 & 22819 & 19823 & 33522\end{array}$

$\begin{array}{lllll}54 & 25075 & 38047 & 75435\end{array}$

$\begin{array}{llllll}58 & 54695 & 63056 & 88644\end{array}$

$\begin{array}{lllll}62 & 12736 & 16879 & 48138\end{array}$

$\begin{array}{lllll}65 & 04509 & 68770 & 92683\end{array}$

$\begin{array}{lllll}67 & 37716 & 16793 & 14191\end{array}$

$\begin{array}{lllll}69 & 20992 & 31811 & 86572\end{array}$

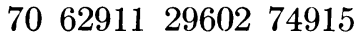

$\begin{array}{lllll}71 & 71374 & 52344 & 73808\end{array}$

$\begin{array}{lllll}72 & 53303 & 45626 & 50671\end{array}$

$\begin{array}{lllll}73 & 14538 & 55406 & 77176\end{array}$

$\begin{array}{lllll}73 & 59867 & 76299 & 09253\end{array}$

$\begin{array}{lllll}73 & 93126 & 40369 & 25937\end{array}$

$\begin{array}{lllll}74 & 17328 & 64517 & 38952\end{array}$

$\begin{array}{lllll}74 & 34805 & 42592 & 48296\end{array}$

$\begin{array}{lllll}74 & 47334 & 40431 & 53499\end{array}$

$\begin{array}{lllll}74 & 56254 & 78400 & 31912\end{array}$

$\begin{array}{lllll}74 & 62564 & 46471 & 11855\end{array}$

$\begin{array}{lllll}74 & 66999 & 66842 & 87939\end{array}$

$\begin{array}{lllll}74 & 70098 & 61871 & 08998\end{array}$

$\begin{array}{lllll}74 & 72251 & 46591 & 09466\end{array}$

$\begin{array}{lllll}74 & 73738 & 78617 & 19263\end{array}$

$\begin{array}{lllll}74 & 74760 & 84919 & 25277\end{array}$

74754595917426951

74759349305142650

$\begin{array}{lllll}74 & 76256 & 74971 & 94430\end{array}$

$\begin{array}{lllll}74 & 76473 \quad 62747 & 36410\end{array}$

$$
q_{r}(49,49)
$$

$\begin{array}{lllll}74 & 76619 & 13284 & 07810\end{array}$

$\begin{array}{lllll}74 & 76716 & 33243 & 94039\end{array}$

$\begin{array}{lllll}74 & 76780 & 99081 & 70684\end{array}$

$\begin{array}{lllll}74 & 76823 & 82642 & 71331\end{array}$

$\begin{array}{lllll}74 & 76852 & 09087 & 92024\end{array}$

$\begin{array}{lllll}74 & 76870 & 66707 & 26980\end{array}$

$\begin{array}{lllll}74 & 76882 & 82803 & 01034\end{array}$

$\begin{array}{lllll}74 & 76890 & 75809 & 13813\end{array}$

$\begin{array}{lllll}74 & 76895 & 90889 & 79998\end{array}$

$\begin{array}{llll}74 & 76899 & 24120 & 51895\end{array}$

74769013883323182 $r$

8

9

10

11

12

13

14

15)

16

17

18

19

20

21

22

23

24

25

26

27

28

29

30

31

32

33

34

35

36

37

38

39

40

41

42

43

44

45

46

47

48

49

$r$

61

62

63

64

6i)

66

67

68

69

70
$P_{r}(49,49)$

$\begin{array}{llll}15 & 87937 & 47277\end{array}$

$\begin{array}{lll}52 & 08652 \quad 47085\end{array}$

1219546210853

$\begin{array}{llll}213 & 7094187417\end{array}$

$292 \quad 2366088026$

$\begin{array}{llll}323 & 71338 & 03249\end{array}$

$\begin{array}{llll}300 & 40681 & 24568\end{array}$

$\begin{array}{llll}240 & 71325 & 68777\end{array}$

$\begin{array}{llll}171 & 08154 & 40544\end{array}$

$\begin{array}{lll}110 & 42127 & 59320\end{array}$

$\begin{array}{llll}66 & 04574 & 73208\end{array}$

$\begin{array}{llll}37 & 23585 & 89548\end{array}$

$\begin{array}{llll}20 & 06417 & 05470\end{array}$

$1044661 \quad 76145$

$\begin{array}{lll}5 & 2994433056\end{array}$

$2 \quad 6351505294$

12897150552 6229344602 2973641660 1403875228

655517660 302658703 138094202 $\begin{array}{ll}622 & 29879\end{array}$ $\begin{array}{lll}276 & 69589\end{array}$ 12130780 5236586 2224235

927622

379693

151958 59521 22652 8406 2998 1043 339 109 31 9 $q_{r}(49,49)$

$\begin{array}{ll}74 & 769027660981020\end{array}$ $7476903 \quad 6464421013$ 74769042065016696 74769045612010320 74 $76904 \quad 78479 \quad 57187$ $\begin{array}{llll}74 & 76904 & 92506 & 52774\end{array}$ $\begin{array}{lllll}74 & 76905 & 01262 & 04927\end{array}$ $\begin{array}{llll}74 & 76905 & 06698 & 67949\end{array}$ $\begin{array}{lllll}74 & 76905 & 10056 & 10432\end{array}$ $\begin{array}{lllll}74 & 76905 & 12117 & 72942\end{array}$ $\begin{array}{lllll}71 & 74 & 76905 & 13376 & 13392\end{array}$ 


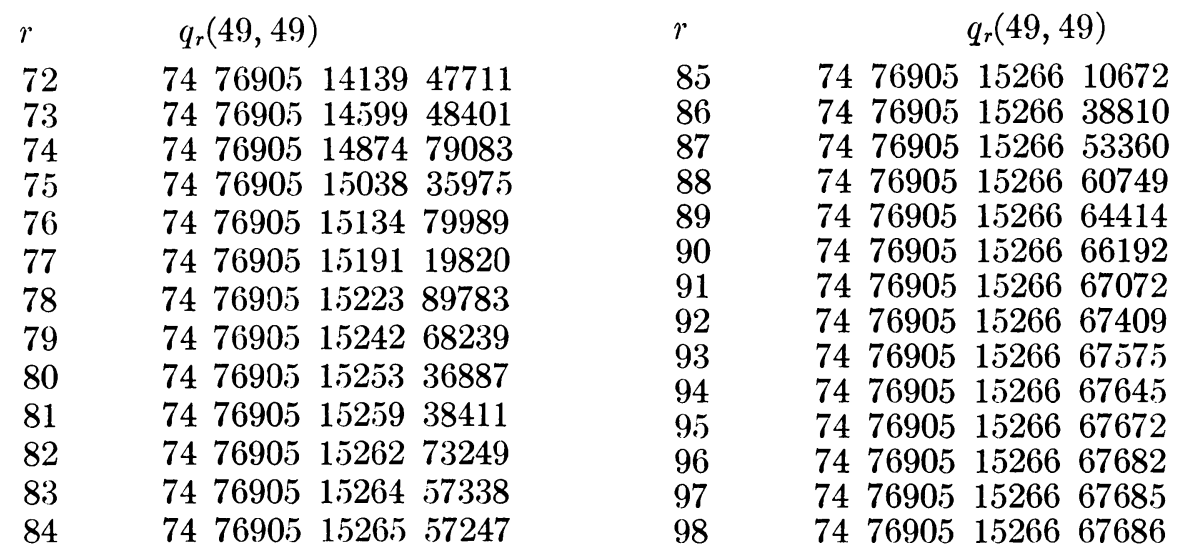

The University of Arizona

Tucson, Arizona

1. B. GoRdon, "Some identities in combinatorial analysis," Quart. J. Math. Oxford Ser. 2, v. 12,1961, p. $285-290$. p. 591 .

2. L. CARlitz, "A note on the Jacobi theta formula," Bull. Amer. Math. Soc., v. 68, 1962,

3. G. Szeneres, "An asymptotic formula in the theory of partitions," Quart. J. Math. Oxford Ser. 2, v. 2, 1951, p. 85-108. 